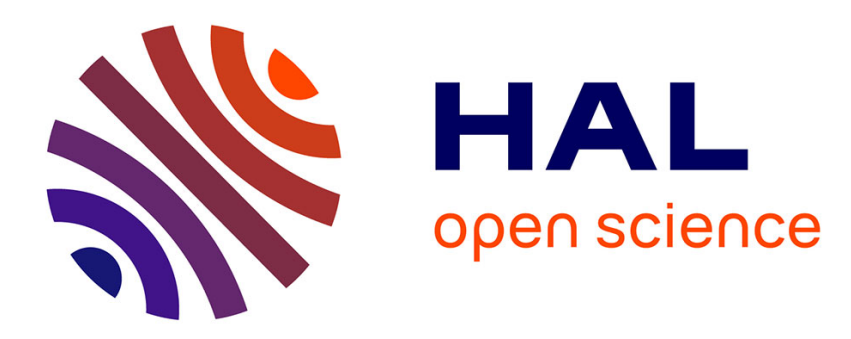

\title{
Fate of Dynamical Many-body Localization in the Presence of Disorder
}

\author{
Analabha Roy, Arnab Das
}

\section{To cite this version:}

Analabha Roy, Arnab Das. Fate of Dynamical Many-body Localization in the Presence of Disorder. Physical Review B: Condensed Matter and Materials Physics (1998-2015), 2015, 91, pp.121106(R). 10.1103/PhysRevB.91.121106 . hal-03019487

\section{HAL Id: hal-03019487 https://hal.science/hal-03019487}

Submitted on 23 Nov 2020

HAL is a multi-disciplinary open access archive for the deposit and dissemination of scientific research documents, whether they are published or not. The documents may come from teaching and research institutions in France or abroad, or from public or private research centers.
L'archive ouverte pluridisciplinaire HAL, est destinée au dépôt et à la diffusion de documents scientifiques de niveau recherche, publiés ou non, émanant des établissements d'enseignement et de recherche français ou étrangers, des laboratoires publics ou privés. 


\title{
Fate of dynamical many-body localization in the presence of disorder
}

\author{
Analabha Roy ${ }^{1}$ and Arnab Das ${ }^{2, *}$ \\ ${ }^{1}$ Saha Institute of Nuclear Physics, 1/AF Bidhannagar, Kolkata-700064, India \\ ${ }^{2}$ Indian Association for the Cultivation of Science, 2A \& 2B Raja S. C. Mullick Road, Kolkata-700032, India \\ (Received 15 September 2014; revised manuscript received 22 February 2015; published 13 March 2015)
}

\begin{abstract}
Dynamical localization is one of the most startling manifestations of quantum interference, where the evolution of a simple system is frozen out under a suitably tuned coherent periodic drive. Here we show that, although any randomness in the interactions of a many-body system kills dynamical localization eventually, spectacular remnants survive even when the disorder is strong. We consider a disordered quantum Ising chain where the transverse magnetization relaxes exponentially with time with a decay time-scale $\tau$ due to random longitudinal interactions between the spins. We show that, under external periodic drive, this relaxation slows down ( $\tau$ shoots up) by orders of magnitude as the ratio of the drive frequency $\omega$ and amplitude $h_{0}$ tends to certain specific values (the freezing condition). If $\omega$ is increased while maintaining the ratio $h_{0} / \omega$ at a fixed freezing value, then $\tau$ diverges exponentially with $\omega$. The results can be easily extended for a larger family of disordered fermionic and bosonic systems.
\end{abstract}

The dynamics of quantum systems driven out of equilibrium by coherent periodic drives has remained an intriguing topic of interest from the early days of quantum mechanics (see, e.g., [1]) to date [2-28]. One interesting and well-known phenomenon in this field, where repeated quantum interference results in a scenario which is quite counterintuitive and unexpected from the classical point of view, is that of dynamical freezing in a quantum system under a periodic drive. Illustrious examples include the localization of a single quantum particle for all time while being forced periodically in free space (dynamical localization [29]), or in one of the two wells of a double-well potential modulated sinusoidally (coherent destruction of tunneling [30]). In both cases, this happens due to the coherent suppression of tunneling.

A many-body version of this phenomenon, dubbed as dynamical many-body freezing, has also been observed both theoretically [31-36] and experimentally [26]. Dynamical many-body freezing, however, is a more drastic version of dynamical localization: In the latter only the tunneling term is renormalized to zero by the external drive, resulting in localization in real space, while in the former the entire many-body Hamiltonian-with all its mutually noncommuting termsvanishes [31]. This implies freezing of any arbitrary initial state in the Hilbert space, rather than freezing of initial states localized in real space only. Intuitively, such an unequivocal freezing of all degrees of freedom of a many-body system seems possible only under very special circumstances, where certain simplicities in the structure of the Hamiltonian allow for such large-scale destructive quantum interference. Here we demonstrate that such dynamical many-body freezing can have strong manifestations even in a system where dynamics is induced by interactions which are totally random.

The plan of the paper is as follows. After introducing the system and the drive, we briefly sketch the content of our analytical approach to the problem. Then we discuss our main results in that light. The precise condition of maximal freezing is obtained from this analysis. We also go beyond

\footnotetext{
*arnab.das.physics@gmail.com
}

that, using exact numerical results for large system sizes, averaged over several disorder realizations, and discuss further characteristics of the phenomenon. Finally we conclude with an outlook.

Consider the following disordered one-dimensional Ising chain, subjected to a sinusoidal transverse field:

$$
H(t)=-\alpha J \sum_{i}^{L-1} J_{i} \sigma_{i}^{x} \sigma_{i+1}^{x}-\sum_{i}^{L}\left\{h_{0} \sin (\omega t)+\alpha h_{i}\right\} \sigma_{i}^{z},
$$

where $\sigma^{\alpha}$ 's $(\alpha=x, y, z)$ are components of Pauli spins, $J_{i}$ and $h_{i}$ are, respectively, the (quenched) interactions and on-site fields - both drawn randomly from a uniform distribution between $(-1,+1)$. The transverse field is subjected to an external drive of frequency $\omega($ period $T=2 \pi / \omega)$ and amplitude $h_{0}$ (we set $\hbar=1)$.

The Hamiltonian in Eq. (1) can be mapped to the Hamiltonian [37-39]

$$
\begin{aligned}
H(t)= & -\alpha J \sum_{i}^{L} J_{i}\left(c_{i}^{\dagger} c_{i+1}^{\dagger}+c_{i}^{\dagger} c_{i+1}+\text { H.c. }\right) \\
& -2 \sum_{i}^{L}\left\{h(t)+\alpha h_{i}\right\} c_{i}^{\dagger} c_{i},
\end{aligned}
$$

with hard-core bosons created (annihilated) by $c_{i}^{\dagger}\left(c_{i}\right)$ These bosons satisfy $\left\{c_{j}^{\dagger}, c_{j}\right\}=0$, and the usual bosonic commutation relations for $i \neq j$. Also, $h(t)=h_{0} \sin \omega t$.

In order to gain insight into the drive-dependent sharp jumps in the relaxation time scale, we follow a recently developed scheme [40] of deducing a renormalized time-independent effective Hamiltonian $H_{\text {eff }}$ which describes the evolution of the system if observed stroboscopically at instants $t=n T$, where $n$ denotes natural numbers: $U(n T)=e^{-i H_{\text {eff }} n T}$, where $U(t)=\mathcal{T} \exp \left[-i \int_{0}^{t} H\left(t^{\prime}\right) d t^{\prime}\right](\mathcal{T}$ denotes time ordering). For $\omega \gg|\alpha J|$, i.e., in the limit of fast drive, no appreciable evolution takes place within a period, and stroboscopic observations represent smooth evolutions to a good approximation. It follows from Floquet theory [41] that for a $T$-periodic 

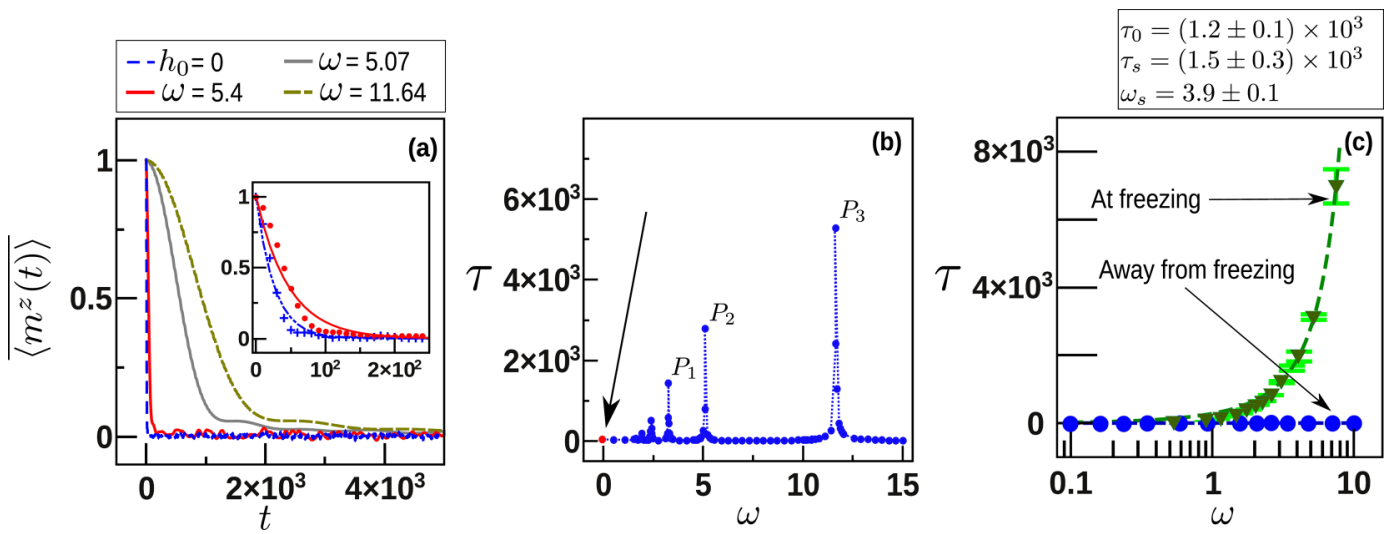

FIG. 1. (Color online) Dynamical freezing without field disorder $\left(h_{i}=0\right.$ ). (a) Exponential relaxation of the expectation value of $m^{z}$ with time for different values of the drive frequencies $\omega$. Unless otherwise indicated, the drive amplitude $h_{0}$ is fixed at 7.0. For certain specific values of $\omega$ (e.g., $\omega=5.07,11.64$ ), the relaxation is tremendously slowed down. The relaxation in the absence of the drive is labeled separately for comparison. The inset compares a representative sample of the numerical data (shown as points) to the curves fitted to them using Eq. (6). (b) $\tau$ vs $\omega$ for fixed $h_{0}$. The sharp peaks indicate dramatic enhancements of $\tau$ for certain values of $\omega$. Three of the most prominent peaks are identified as $P_{1-3}$. The values of $\omega$ at these peaks are $P_{1} \approx 3.24, P_{2} \approx 5.07$, and $P_{3} \approx 11.64$. Those values are identified to be the ones for which the effective Hamiltonian $H_{\text {eff }}$ [Eq. (4)] vanishes. The red dot pointed by the arrowhead represents the case in absence of the drive. (c) $\tau$ vs $\omega$ at fixed $\eta$. Comparison of enhancement of $\tau$ as $\omega$ is increased keeping $\eta=\frac{4 h_{0}}{\omega}$ fixed for two cases-under the freezing condition $\mathcal{J}_{0}(\eta)=0(\eta \approx 2.4048)$, and away from it $\mathcal{J}_{0}(\eta) \approx 0.765(\eta=1.0)$ as marked in the figure. Exponential enhancement of $\tau$ with $\omega$ is observed (numerical data fitted with the $\left.\tau(\omega)\right|_{\mathcal{J}_{0}(\eta)=0}=\tau_{0}+\tau_{s} e^{\omega / \omega_{s}}$ form) under the freezing condition, while no noticeable variation of $\tau$ is observed away from the freezing condition. Results are for $L=100$, averaged over $>10^{3}$ disorder realizations of the bonds $J_{i}$. The error bars due to disorder-induced fluctuations are about the point size (see, e.g., Fig. S1 of the Supplemental Material [44]), and hence omitted [except for (c)] to avoid cluttering.

Hamiltonian $H(t)$, the time-evolution operator can be written as $U(t)=e^{-i H_{\text {eff }} t} Z(t)$, where $Z(t)$ is $T$ periodic and $H_{\text {eff }}$ is Hermitian. Clearly $H_{\text {eff }}$ is an operator that is largely nonlocal in the original degrees of freedom and is not necessarily amenable to any simple physical interpretation. However, since $Z(0)=Z(n T)=\mathbb{1}$ (identity), $H_{\text {eff }}$ can be considered as an effective time-independent Hamiltonian giving the correct stroboscopic evolution. Using the above decomposition of $U(t)$ and the time-dependent Schrödinger equation it satisfies, we get

$$
H_{\mathrm{eff}}=Z^{\dagger}(t) H(t) Z(t)-i Z^{\dagger}(t)\left[\partial_{t} Z(t)\right] .
$$

Deducing the exact form of $H_{\text {eff }}$ from the above equation is as hard as solving the original time-dependent problem. However, controlled approximations in the large $\omega$ limit can be obtained [40] using a flow equation technique $[42,43]$ which we employ here. This yields (see Supplemental Material for details [44])

$$
\begin{aligned}
H_{\mathrm{eff}}= & -J \sum_{i} j_{i}^{(0)}\left(c_{i}^{\dagger} c_{i+1}^{\dagger}+\text { H.c. }\right) \\
& -J \sum_{i} j_{i}^{(1)}\left(c_{i}^{\dagger} c_{i+1}+\text { H.c }\right)-\mu \sum_{\mathrm{i}} \mathrm{j}_{\mathrm{i}}^{(2)} \mathrm{c}_{\mathrm{i}}^{\dagger} \mathrm{c}_{\mathrm{i}},
\end{aligned}
$$

with $\eta \equiv 4 h_{0} / \omega$, and the constants $j_{i}^{(s)}, \mu$ defined as follows:

$$
\begin{aligned}
j_{i}^{(0)} & \equiv \alpha J_{i}\left\{\mathcal{J}_{0}(\eta)-\frac{4 \alpha h_{i}}{\omega} \beta(\eta)\right\}, \quad j_{i}^{(1)} \equiv \alpha J_{i} \mathcal{J}_{0}(\eta), \\
j_{i}^{(2)} & \equiv \frac{h_{i}}{J}, \quad \mu \equiv 2 \alpha J .
\end{aligned}
$$

Here $\mathcal{J}_{0}(\eta)$ is the ordinary Bessel function of order zero [note that the way they are defined in Eq. (1), $J_{i}$ are dimensionless and $h_{i}$ have dimension of energy]. In addition, the function $\beta(\eta) \equiv \sum_{n \neq 0} \mathcal{J}_{n}(\eta) / n$.

The above expression for $H_{\text {eff }}$ is obtained under a rotatingwave approximation (RWA) which holds for $\omega \gg J, \alpha$. This effective Hamiltonian accurately reproduces the dynamics of the full system stroboscopically to the lowest order in $\alpha / \omega$ [44].

Now we describe our main results for the driven spin Hamiltonian $H(t)$ and analyze it in light of the effective time-independent Hamiltonian $H_{\text {eff }}$. To avoid cluttering, the results presented in Fig. 1 and the main discussion are focused on the case where the field disorder is absent $\left(h_{i}=0\right)$, the case with $h_{i} \neq 0$ is given afterwards. Imagine that the spins [in $H(t)$ ] are initially prepared in a state strongly polarized in the $+z$ direction and the transverse magnetization $m^{z}=$ $\frac{1}{L}\left\langle\sum_{i=1}^{L} \sigma_{i}^{z}\right\rangle \approx 1$. Here $\langle\cdots\rangle$ denotes quantum expectation values, and $\bar{O}$ denotes average of $O$ over disorder realizations. In the absence of the drive $\left(h_{0}=0\right)$, the magnetization $m^{z}$ relaxes with time since the random interaction terms in the Hamiltonian do not commute with it. If the drive is switched on, the characteristic time scale of the relaxation changes, depending on the drive. The relaxations of $\overline{m^{z}}$ with time for various drive frequencies are shown in Fig. 1(a) (these results are obtained by numerically solving the time-dependent Schrödinger equation for several disorder realizations and averaging over them). The relaxations (both in the absence and presence of the drive) are fitted accurately with the exponential decay form [inset of Fig. 1(a)]

$$
\overline{\left\langle m^{z}(t)\right\rangle}=m_{0}^{z} e^{-t / \tau},
$$

where $\tau$ is the decay constant. Our main result concerns the behavior of the decay time-scale $\tau$ as a function of the drive frequency $\omega$ for a given drive amplitude $h_{0}$. 


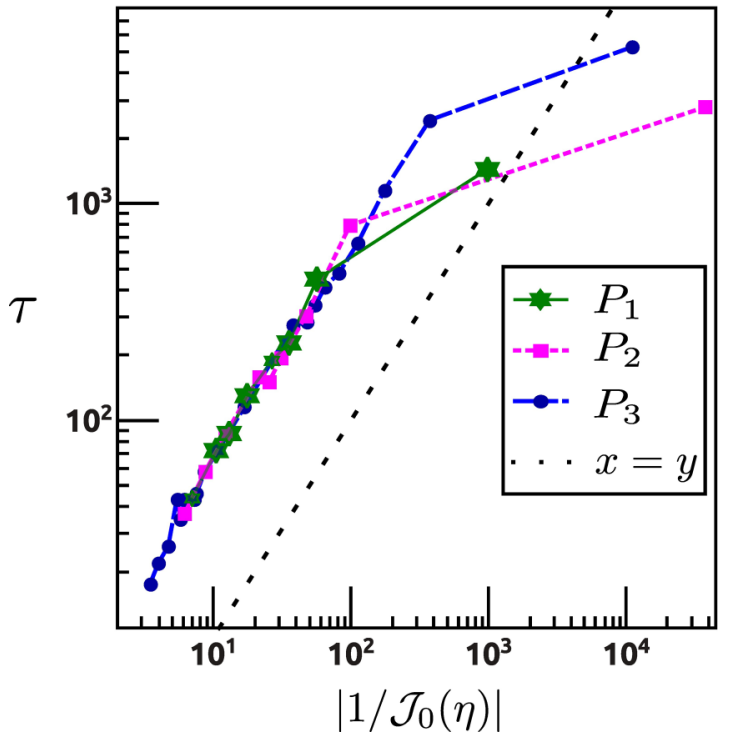

FIG. 2. (Color online) Dependence of $\tau$ on the drive parameters. Numerical data for $\tau$ vs $1 / \mathcal{J}_{0}(\eta)$ for $h_{i}=0, h_{0}=7$ (for a single random realization) are shown for the range of $\omega$ around the peaks $P_{1,2,3}$ of Fig. 1(b). The black dashed line is a guide to the eye. As expected from Eqs. (4) and (5), we observe $\tau \propto 1 / \mathcal{J}_{0}(\eta)$, where $\mathcal{J}_{0}(\eta) \gg \alpha / \omega^{2}$. However, for small $\mathcal{J}_{0}(\eta), \tau$ tends to saturate to a large value viz. the respective peak values in Fig. 1(b) (instead of diverging).

Freezing points. The behavior is quite dramatic, as shown in Fig. 1(b): for certain values of $\omega$, the value of $\tau$ shoots up sharply by orders of magnitude compared to the undriven case. This corresponds to the extreme slowing down (freezing) of the decay of $m^{z}$ visible for certain values of $\omega$ in Fig. 1(a). This can be explained by noting that for $h_{i}=0$ (as considered in the figure), the effective Hamiltonian $H_{\text {eff }} \propto \mathcal{J}_{0}(\eta)$ to leading order in $\alpha / \omega$ [Eqs. (4) and (5)] and hence $\tau \propto 1 / \mathcal{J}_{0}(\eta)$ for $\omega \gg$ $J, \alpha$ (see Fig. 2). Thus, there is an overall renormalization of the time scale, which can be controlled by the factor $\mathcal{J}_{0}(\eta)$. Under the special condition $\mathcal{J}_{0}(\eta)=0$ (the freezing condition), $H_{\text {eff }}$ vanishes, indicating a large enhancement of all time scales observable in the dynamics governed by $H_{\text {eff }}$. Interestingly, this implies that the slowing down is not only limited to $m^{z}(t)$, or to any special initial condition. For finite $\omega$, the approximation is, of course, valid only as long as $\mathcal{J}_{0}(\eta) \gg \alpha / \omega^{2}$, otherwise higher order terms in $\alpha / \omega$ come into play. This results in a saturation due to the breakdown in the linear behavior of $\tau$ with $1 / \mathcal{J}_{0}(\eta)$ (Fig. 2). The saturation is also seen in the large but finite values of $\tau$ observed at the freezing points $\left(P_{1,2,3}\right)$ of Fig. 1(b), instead of infinite $\tau$ as suggested by the vanishing of $H_{\mathrm{eff}}$ at those points.

Beyond the rotating-wave approximation. Though $\tau$ does not diverge for finite $\omega$ due to higher order corrections to RWA, those corrections should vanish as $\omega \rightarrow \infty$, resulting in $\lim _{\omega \rightarrow \infty} \tau \rightarrow \infty$ under the freezing condition $\mathcal{J}_{0}(\eta)=$ 0 . To characterize the dependence of $\tau$ on $\omega$ under the freezing condition going beyond RWA, we numerically study the variation of $\tau$ with $\omega$ fixing $\eta$ to a freezing value. Figure 1(c) shows that $\tau$ diverges exponentially with $\omega$. The numerical results in the figure are fitted well with the form
$\left.\tau(\omega)\right|_{\mathcal{J}_{0}(\eta)=0}=\tau_{s} e^{\omega / \omega_{s}}-\tau_{0}$. If $\eta$ is held fixed to some value such that $\mathcal{J}_{0}(\eta) \not \approx 0$ (away from freezing), $\tau$ does not show any appreciable change with increase in $\omega$ within the range considered [Fig. 1(c)]. In this range, $\tau$ increased by orders of magnitude for the freezing case.

The $\omega \rightarrow \infty$ limit. Note that absolute freezing, i.e., the divergence of $\tau$ in the $\omega \rightarrow \infty$ limit under the freezing condition, is a counterintuitive result. Intuitively, an infinitely fast purely sinusoidal drive should simply be equivalent to the absence of the drive altogether, since the driven parameters return back to themselves within no appreciable time in each cycle. This would mean that $m^{z}$ should decay when $\omega \rightarrow \infty$ as if there was no drive at all. This is indeed the case away from freezing - in the large $\omega$ range we considered [see Fig 1(c)] $\tau$ remains the same as that of the undriven case as $\omega$ is increased. However, under the freezing condition, $\tau$ diverges exponentially, indicating that the decay will completely stop due to the drive as $\omega \rightarrow \infty$.

Static disordered field $\left(h_{i} \neq 0\right)$. Now we briefly present the results for the case with quenched disorder in the transverse field. Including randomness in the field does not alter the basic features of the phenomenon discussed above, but there are some quantitative differences that we discuss here. In this case $H_{\text {eff }}$ does not vanish even to first order in $1 / \omega$ at the freezing point due to the field-dependent terms in Eq. (4) that scale inversely with $\omega$. Thus, we expect freezing at the roots of $\mathcal{J}_{0}(\eta)$ to last for shorter times at large $\omega$ when the field disorder is on. This is qualitatively verified by our numerical simulations of the exact dynamics with field disorder on. Figure 3 shows plots of $m^{z}$ and the exponential relaxation time scale therein in regimes similar to Fig. 1, except with the field disorder switched on. The plots show that freezing is maintained at the roots of $\mathcal{J}_{0}(\eta)$, although the time scale of the decay is one order of magnitude less than the case without any static field.

Summary and outlook. We have shown that dynamical localization can have drastic manifestations in many-body systems with extensive disorder. We show that the application of a coherent periodic drive with specific values of the ratio of the drive frequency $\omega$ and the amplitude $h_{0}$ (freezing condition) can drastically slow down the natural relaxation induced by random interactions between the spins in a disordered Ising chain for any arbitrary initial state in the Hilbert space. For moderately high values of $\omega$ and $h_{0}, \tau$ is observed to be orders of magnitude higher than its value away from freezing (or that in absence of the drive). At a specific freezing point ( $h_{0} / \omega$ kept fixed), the characteristic relaxation time $\tau$ diverges exponentially with $\omega$. However, if the ratio is fixed away from the freezing value, increasing $\omega$ does not have any observable effect on $\tau$. These results are the first of their kind, showing drastic survival of dynamical localization, which is the result of large-scale destructive quantum interference induced by a periodic drive, in a highly disordered system. This opens up possibilities of preserving arbitrary (even unknown) quantum states of interacting qubits (Ising spins) from decaying due to unknown stray interactions. Experimental realizations of bosons in disordered 1D potentials in optical lattices have already been achieved experimentally $[45,46]$. Coherent periodic drives applied to hardcore bosons in optical lattices, used for realizing spin chains with precise control over the Ising-like couplings, have also materialized 

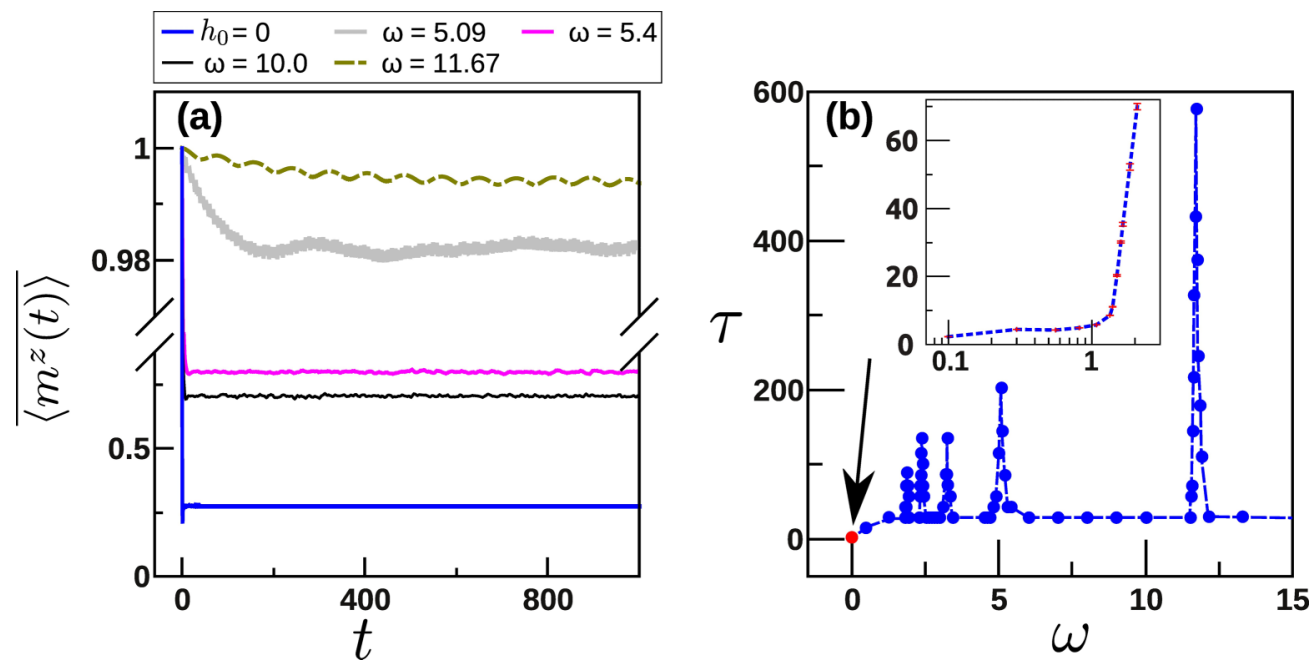

FIG. 3. (Color online) Dynamical freezing with field disorder. (a) Exponential relaxation of $m^{z}$ with time for different values of the drive frequencies $\omega$. (b) The time-scale $\tau$ of the exponential relaxation of $m^{z}$ vs $\omega$. Note the peaks at the roots of $\mathcal{J}_{0}(\eta)$, the same places as peaks $P_{1-3}$ in Fig 1. The red dot pointed by the arrowhead represents the case in absence of the drive. The inset plots $\tau$ vs $\omega$ (log-scale) at smaller values while adjusting $h_{0}$ so as to remain at the first root of $\mathcal{J}_{0}(\eta)$. At very small values of $\omega$, the higher order contributions destroy the dynamical equivalence between $H(t)$ and $H_{\text {eff }}$, leading to a loss of freezing. Freezing begins to appear as $\omega$ is increased, as seen in the inset by the growth of $\tau$ with $\omega$ for $\eta$ fixed to a freezing value. All the parameters are the same as in Fig 1, except with field disorder $h_{i}$ switched on. The $h_{i}$ are distributed uniformly and randomly between \pm 1 .

[24]. Hence, experimental observations of the freezing phenomenon seem feasible in a present day cold-atom laboratory.

Acknowledgments. A.R. thanks the Council of Scientific and Industrial Research, India, for support under Scientists'
Pool Scheme No. 13(8531-A)/2011/Pool. Computational resources were provided by the clusters at the Texas Advanced Computational Center, University of Texas at Austin. Both authors thank Kasturi Basu, IACS Kolkata, for useful discussions.
[1] J. J. Sakurai, Modern Quantum Mechanics, revised ed. (Addison-Wesley, Reading, MA, 1994).

[2] A. Eckardt, C. Weiss, and M. Holthaus, Superfluid-insulator transition in a periodically driven optical lattice, Phys. Rev. Lett. 95, 260404 (2005).

[3] A. Eckardt and M. Holthaus, Avoided-level-crossing spectroscopy with dressed matter Waves, Phys. Rev. Lett. 101, 245302 (2008).

[4] A. Lazarides, A. Das, and R. Moessner, Periodic thermodynamics of isolated quantum systems, Phys. Rev. Lett. 112, 150401 (2014).

[5] A. Lazarides, A. Das, and R. Moessner, Equilibrium states of generic quantum systems subject to periodic driving, Phys. Rev. E 90, 012110 (2014).

[6] E. Arimondo, D. Ciampini, A. Eckardt, M. Holthaus, and O. Morsch, Advances in Atomic, Molecular, and Optical Physics (Academic, New York, 2012), Vol. 61.

[7] J. Struck, C. Ölschläger, M. Weinberg, P. Hauke, J. Simonet, A. Eckardt, M. Lewenstein, K. Sengstock, and P. Windpassinger, Tunable gauge potential for neutral and spinless particles in driven optical lattices, Phys. Rev. Lett. 108, 225304 (2012).

[8] P. Hauke et al., Non-Abelian gauge fields and topological insulators in shaken optical lattices, Phys. Rev. Lett. 109, 145301 (2012).

[9] N. H. Lindner, G. Refael, and V. Galitski, Floquet topological insulator in semiconductor quantum wells, Nat. Phys. 7, 490 (2011).
[10] M. Thakurathi, A. A. Patel, D. Sen, and A. Dutta, Floquet generation of Majorana end modes and topological invariants, Phys. Rev. B 88, 155133 (2013).

[11] T. Prosen and E. Ilievski, Nonequilibrium phase transition in a periodically driven $X Y$ spin chain, Phys. Rev. Lett. 107, 060403 (2011).

[12] V. M. Bastidas, C. Emary, G. Schaller, and T. Brandes, Nonequilibrium quantum phase transitions in the Ising model, Phys. Rev. A 86, 063627 (2012).

[13] V. Mukherjee and A. Dutta, Effects of interference in the dynamics of a spin- $1 / 2$ transverse $X Y$ chain driven periodically through quantum critical points, J. Stat. Mech. (2009) P05005.

[14] T. Nag, S. Roy, A. Dutta, and D. Sen, Dynamical localization in a chain of hard core bosons under periodic driving, Phys. Rev. B. 89, 165425 (2014).

[15] A. Roy and L. E. Reichl, Coherent control of trapped bosons, Phys. Rev. A 77, 033418 (2008).

[16] A. Roy and L. Reichl, Quantum control of interacting bosons in periodic optical lattice, Physica E 42, 1627 (2010).

[17] A. Das, K. Sengupta, D. Sen, and B. K. Chakrabarti, Infiniterange Ising ferromagnet in a time-dependent transverse magnetic field: Quench and ac dynamics near the quantum critical point, Phys. Rev. B 74, 144423 (2006).

[18] S. Mondal, D. Pekker, and K. Sengupta, Dynamics-induced freezing of strongly correlated ultracold bosons, Europhys. Lett. 100, 60007 (2012) 
[19] S. Ashhab, J. R. Johansson, A. M. Zagoskin, and F. Nori, Twolevel systems driven by large-amplitude fields, Phys. Rev. A 75, 063414 (2007).

[20] L. D'Alessio and M. Rigol, Long-time behavior of isolated periodically driven interacting lattice systems, Phys. Rev. X 4, 041048 (2014).

[21] P. Ponte, A. Chandran, Z. Papic, and D. A. Abanin, Periodically driven ergodic and many-body localized quantum systems, Ann. Phys. 353, 196 (2015).

[22] H. Lignier, C. Sias, D. Ciampini, Y. Singh, A. Zenesini, O. Morsch, and E. Arimondo, Dynamical control of matter-wave tunneling in periodic potentials, Phys. Rev. Lett. 99, 220403 (2007).

[23] A. Zenesini, H. Lignier, D. Ciampini, O. Morsch, and E. Arimondo, Coherent control of dressed matter waves, Phys. Rev. Lett. 102, 100403 (2009).

[24] Y.-A. Chen, S. Nascimbène, M. Aidelsburger, M. Atala, S. Trotzky, and I. Bloch, Controlling correlated tunneling and superexchange interactions with ac-driven optical lattices, Phys. Rev. Lett. 107, 210405 (2011).

[25] J. Struck et al., Quantum simulation of frustrated classical magnetism in triangular optical lattices, Science 333, 996 (2011).

[26] S. Hegde, H. Katiyar, T. S. Mahesh, and A. Das, Freezing a quantum magnet by repeated quantum interference: An experimental realization, Phys. Rev. B 90, 174407 (2014).

[27] F. J. Kaiser, P. Hänggi, and S. Kohler, Molecular electronics in junctions with energy disorder, New J. Phys. 10, 065013 (2008).

[28] V. A. Gopar and R. A. Molina, Controlling conductance statistics of quantum wires by driving ac fields, Phys. Rev. B 81, 195415 (2010).

[29] D. H. Dunlap and V. M. Kenkre, Dynamic localization of a charged particle moving under the influence of an electric field, Phys. Rev. B 34, 3625 (1986).

[30] F. Grossmann, T. Dittrich, P. Jung, and P. Hänggi, Coherent destruction of tunneling, Phys. Rev. Lett. 67, 516 (1991).

[31] A. Das, Exotic freezing of response in a quantum many-body system, Phys. Rev. B 82, 172402 (2010).

[32] S. Bhattacharyya, A. Das, and S. Dasgupta, Transverse Ising chain under periodic instantaneous quenches: Dynamical many- body freezing and emergence of slow solitary oscillations, Phys. Rev. B 86, 054410 (2012).

[33] A. Das and R. Moessner, Switching the anomalous DC response of an AC-driven quantum many-body system, arXiv:1208.0217.

[34] A. Russomanno, A. Silva, and G. E. Santoro, Periodic steady regime and interference in a periodically driven quantum system, Phys. Rev. Lett. 109, 257201 (2012).

[35] M. Bukov, L. D’Alessio, and A. Polkovnikov, Universal highfrequency behavior of periodically driven systems: From dynamical stabilization to floquet engineering, arXiv:1407.4803.

[36] S. Suzuki, J. Inoue, and B. K. Chakrabarti, Quantum Ising Phases and Transitions in Transverse Ising Models, Lecture Notes in Physics, Vol. 862 (Springer, Berlin, 2013).

[37] E. Lieb, T. Schultz, and D. Mattis, Two soluble models of an antiferromagnetic chain, Ann. Phys. 16, 407 (1961).

[38] A. P. Young and H. Rieger, Numerical study of the random transverse-field Ising spin chain, Phys. Rev. B 53, 8486 (1996).

[39] J. Dziarmaga, Dynamics of a quantum phase transition in the random Ising model: Logarithmic dependence of the defect density on the transition rate, Phys. Rev. B 74, 064416 (2006).

[40] A. Verdeny, A. Mielke, and F. Mintert, Accurate effective Hamiltonians via unitary flow in Floquet space, Phys. Rev. Lett. 111, 175301 (2013).

[41] G. Floquet, Sur les équations différentielles linéaires à coefficients périodiques, Ann. Ecole Norm. Sup. 12, 47 (1883).

[42] F. Wegner, Flow-equation for Hamiltonians, Ann. Phys. 3, 77 (1994).

[43] S. Kehrein, The Flow Equation Approach to Many-Particle Systems (Springer, Berlin, 2006).

[44] See supplemental material at http://link.aps.org/supplemental/ 10.1103/PhysRevB.91.121106 for details on the numerical methodology used to simulate the Schroedinger dynamics of the exact Hamiltonian in Eq. (2), as well as the analytical RG formalism used to derive the expression for $H_{\text {eff }}$ in Eq. (4). Additional plots for the disorder fluctuations and analyticalnumerical comparisons are also provided.

[45] J. E. Lye, L. Fallani, M. Modugno, D. S. Wiersma, C. Fort, and M. Inguscio, Bose-Einstein condensate in a random potential, Phys. Rev. Lett. 95, 070401 (2005).

[46] I. Bloch, Ultracold quantum gases in optical lattices, Nat. Phys. 1, 23 (2005). 